\title{
Transmission patterns of human enterovirus 71 to, from and among European countries, 2003 to 2013
}

C Hassel $^{1}$, A Mirand ${ }^{12}$, A Lukashev ${ }^{3}$, E TerletskaiaLadwig ${ }^{4}$, A Farkas ${ }^{5}$, I Schuffenecker ${ }^{6}$, S Diedrich ${ }^{7}$, HP Huemer ${ }^{8}$, C Archimbaud $^{12}$, H Peigue-Lafeuille ${ }^{12}$, C Henquell ${ }^{12}$, J Bailly ${ }^{12}$

1. Clermont Université, Université d’Auvergne, EPIE, EA 4843, Clermont-Ferrand, France

2. CHU Clermont-Ferrand, Service de Virologie, Centre National de Référence des Entérovirus et Paréchovirus - Laboratoire associé, Clermont-Ferrand, France

3. Chumakov Institute of Poliomyelitis and Viral Encephalitides, Moscow, Russia

4. Prof. Gisela Enders \& Kollegen MVZ GbR and Institute of Virology, Infectious Diseases and Epidemiology, Stuttgart, Germany

5. Division of Virology, National Center for Epidemiology, Budapest, Hungary

6. Laboratoire de Virologie Est des Hospices Civils de Lyon, Centre National de Référence des Entérovirus et Paréchovirus, Bron, France

7. National Reference Center for Poliomyelitis and Enterovirus, Robert Koch Institute, Berlin, Germany

8. Austrian Agency for Health and Food Safety, Vienna, Austria

9. Université Claude Bernard, EA4610 VIRPATH, Lyon, France

Correspondence: Jean-Luc Bailly (j-luc.bailly@udamail.fr)

Citation style for this article:
Hassel C, Mirand A, Lukashev A, TerletskaiaLadwig E, Farkas A, Schuffenecker I, Diedrich S, Huemer HP, Archimbaud C, Peigue-Lafeuille Hélène, Henquell Cécile, Bailly J-L. Transmission patterns of human enterovirus 71 to, from and among European countries, 2003 to 2013. Euro Surveill. 2015;20(34):pii=30005. DOI: http:// dx.doi.org/10.2807/1560-7917.ES.2015.20.34.30005

Enterovirus 71 (EV-71) is involved in epidemics of hand, foot, and mouth disease (HFMD) and has been reported to occur with severe neurological complications in eastern and south-east Asia. In other geographical areas, the transmission of this virus is poorly understood. We used large sequence datasets (of the gene encoding the viral protein 1, VP1) and a Bayesian phylogenetic approach to compare the molecular epidemiology and geographical spread patterns of EV-71 subgenogroups $\mathrm{B}_{4}, \mathrm{~B}_{5}, \mathrm{C}_{1}, \mathrm{C}_{2}$, and $\mathrm{C}_{4}$ in Europe relative to other parts of the world. For the study, European countries considered were European Union (EU) Member States and Iceland, Norway and Switzerland. Viruses of the B4, $\mathrm{B}_{5}$, and $\mathrm{C}_{4}$ subgenogroups circulate mainly in eastern and south-east Asia. In Europe sporadic introductions of these subgenogroups are observed, however $\mathrm{C}_{1}$ and $C_{2}$ viruses predominate. The phylogenies showed evidence of multiple events of spread involving $C_{1}$ and $C_{2}$ viruses within Europe since the mid-1990s. Two waves of sporadic C2 infections also occurred in 2010 and 2013. The 2007 Dutch outbreak caused by $C_{2}$ and the occurrence of $\mathrm{B}_{5}$ and $\mathrm{C}_{4}$ infections in the $\mathrm{EU}$ between 2004 and 2013 arose while the circulation of $C_{1}$ viruses was low. A transmission chain involving a $\mathrm{C}_{4}$ virus was traced from Japan to the EU and then further to Canada between 2001 and 2006. Recent events whereby spread of viruses have occurred from, to, and within Europe appear to be involved in the long term survival of EV-71, highlighting the need for enhanced surveillance of this virus.

\section{Introduction}

The results of infections by enterovirus 71 (EV-71) range from the absence of symptoms to acute manifestations, including hand, foot, and mouth disease (HFMD) as well as neurological conditions such as acute meningitis, encephalitis, and poliomyelitis-like disease [1]. The outbreaks caused by this virus since the late $1990 \mathrm{~s}$ pose serious threats to public health in countries of eastern and south-east Asia (http://www.wpro.who. int/emerging_diseases/HFMD/en/) because a number of infections are involved in cardiopulmonary failure and neurological diseases which, in infancy ( $<1$ yearold), can lead to death [2]. During the HFMD epidemics in China between 2010 and 2012, there were 1,737 laboratory-confirmed deaths, of which $93 \%$ were associated with EV-71 infections [3]. The occurrence of fatalities in Asia, notably in Cambodia, China, Malaysia, and Vietnam does not necessarily indicate that the EV-71 variants and strains circulating in these countries have a greater virulence [4]. Rather, this probably reflects the high total number of infections (HFMD cases and asymptomatic infections). The overall disease burden caused by EV-71 led health authorities to develop surveillance systems in parts of eastern and south-east Asia. The World Health Organization provides a guide to clinical management and outbreak prevention of EV-71 infection (http://www.wpro.who.int/emerging_ diseases/documents/HFMDGuidance/en/), for which five inactivated EV-71 vaccine candidates are being evaluated in clinical trials [5].

Unlike for some areas in eastern and south-east Asia, there is currently no particular epidemiological 
Comparison of phylogenies of five enterovirus 71 subgenogroups (B4, B5, C1, C2, C4) with indications of geographical origins of sample sequences, $1986-2013$ ( $\mathrm{n}=675$ sequences)
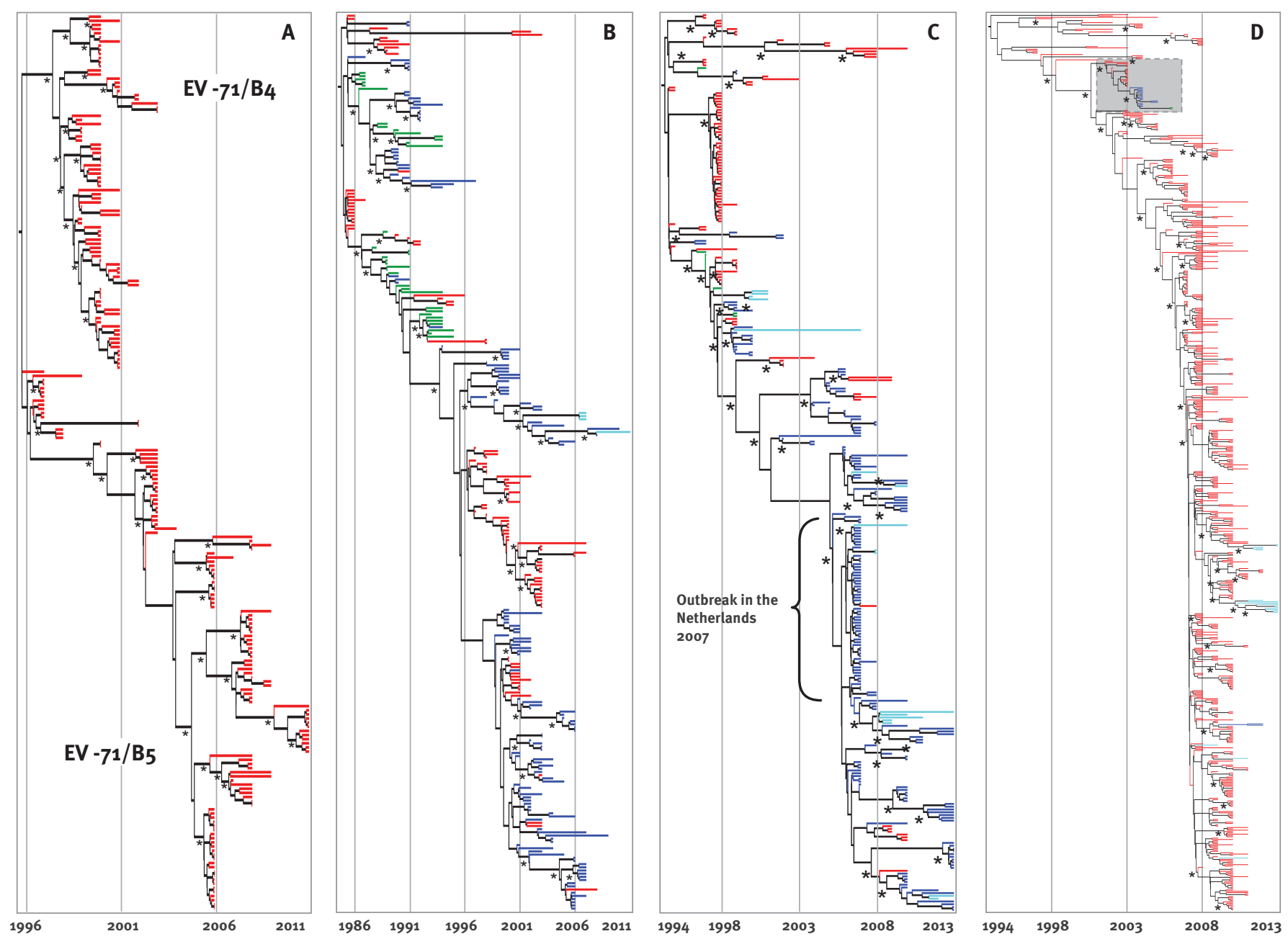

The chronogram trees were inferred with the $1 \mathrm{D}$ gene encoding the capsid viral protein 1 sequence datasets for subgenogroups $\mathrm{B}_{4} / \mathrm{B}_{5}$ (panel A), C1 (panel B), C2 (panel C), and C4 (panel D).

The tree topologies show the genetic relationships between taxa sampled over the periods indicated on the $\mathrm{x}$-axis (calendar years). The phylogenetic relationships were inferred with a Bayesian method using a relaxed molecular-clock model.

For clarity, the sequence names are not included in the tree. Asterisks indicate key nodes with posterior probability (pp) density values >0.90. Each branch tip represents a sampled virus sequence.

The branches in the genealogies of each subgenogroup were coloured to investigate relationships between phylogenetic clustering and the geographical origins of taxa. The geographical areas where the virus strains were sampled are indicated by different colours: blue, Europe (including European Union Member States, Iceland, Norway and Switzerland); light blue, Azerbaijan, Georgia, Kazakhstan, Kyrgyzstan Russia and Ukraine; green, North America; and red, Australia and eastern and south-east Asia.

surveillance of EV-71 infections in the European Union (EU). The virus was involved in outbreaks of neurological diseases in the 1970s in Sweden (1973), Bulgaria (1975; 44 fatalities), Hungary (1978; 47 fatalities), and France (1979) [6-9]. Sero-epidemiological surveys conducted on samples from 1999 to 2007 in Germany showed that ca $12 \%$ of children aged $<5$ years, and ca 40 to $60 \%$ of adults develop neutralising antibodies against EV-71, which suggests that mild and mostly undiagnosed EV-71 infections occur in the general population $[10,11]$. We and others showed that acute EV-71 infections are regularly investigated in patients admitted to hospital in a number of European countries, such as Austria, France, Germany and the Netherlands
[12-14]. Severe manifestations have been occasionally reported in France $[15,16]$, and most documented EV-71 infections in EU Member States included sporadic cases of febrile illness and acute meningitis $[17,18]$.

EV-71 is one serotype among a hundred human enteroviruses (EVs) that belongs to the Picornaviridae family. Virus strains can be divided into six genogroups designated $A$ to $F[19]$ but since the mid-1960s only genogroups $B$ and $C$ have been reported in outbreaks and individual cases of infection. The virus strains of the two major genogroups are classified into subgenogroups $B o$ to $B_{5}$ and $C_{1}$ to $C_{5}$ on the basis of genetic relationships [20]. Subgenogroups $\mathrm{B}_{4}, \mathrm{~B}_{5}$, and $\mathrm{C}_{4}$ 


\section{FIGURE 2}

Plots of genetic diversity ${ }^{\mathrm{a}}$ over time (calendar years) reconstructed for the enterovirus 71 subgenogroups C1 (panel A), C2 (panel B), and C1/C2 (panel C), 1985-2013

A.

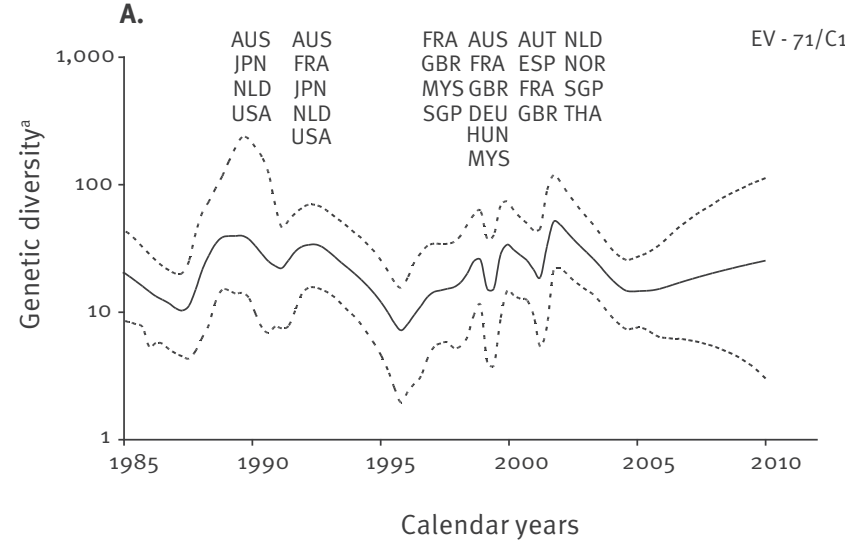

- Effective number of infections'mean $95 \%$ highest probability density interval limit
B.

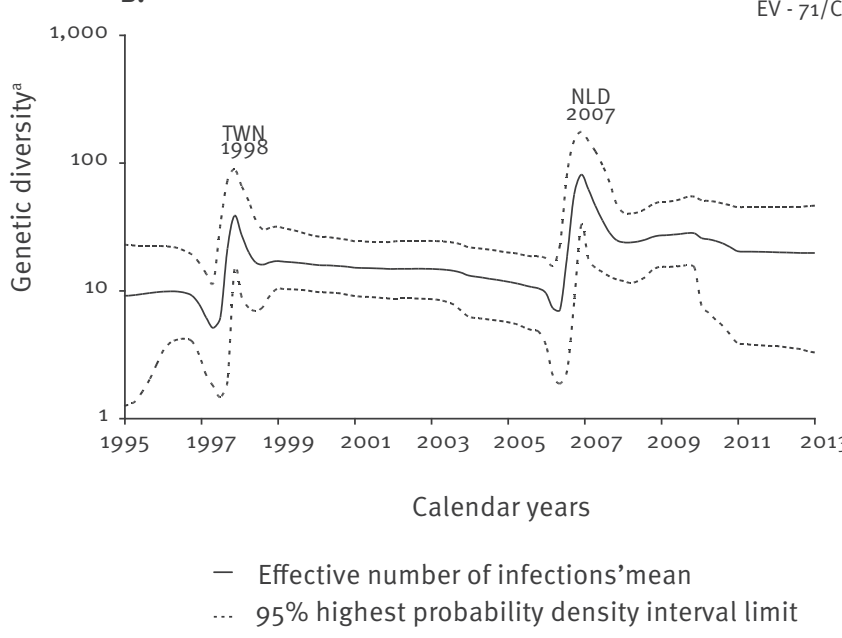

C.

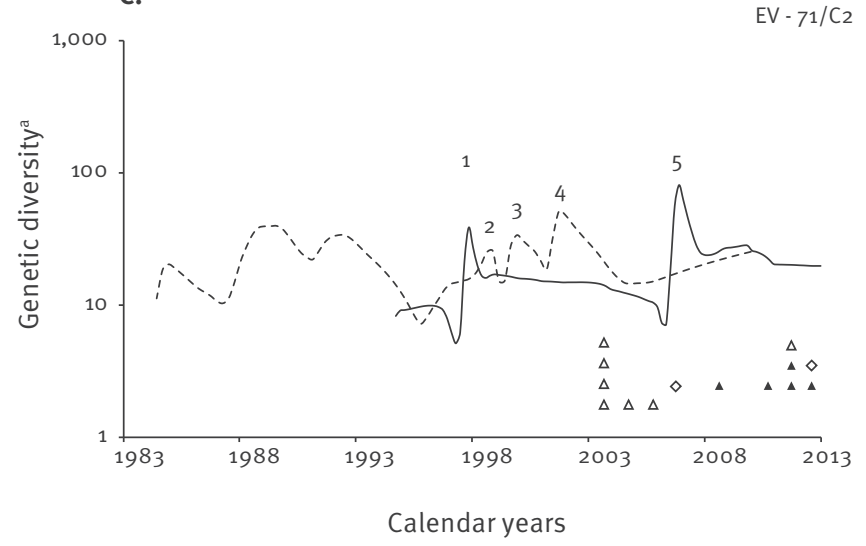

$-\mathrm{EV}-71 / \mathrm{C}_{1} \quad \ldots \mathrm{EV}-71 / \mathrm{C}_{2}$

AUS: Australia; AUT: Austria; DEU: Germany; ESP: Spain; FRA: France; GBR: United Kingdom; HUN: Hungary; JPN: Japan; MYS: Malaysia; NLD: the Netherlands; NOR: Norway; SGP: Singapore; THA: Thailand; TWN: Taiwan; USA: United states.

a Global genetic diversity over time was estimated with gene $1 D$ encoding the capsid viral protein 1 ( $1 D^{\mathrm{VP}_{1}}$ ) for each subgenogroup. Genetic diversity is estimated with the Bayesian skyline plot model and is expressed as log1oNet (Ne: effective size of virus population; t: generation time). Genetic diversity reflects the effective number of infections averaged over time under the assumption of a neutral evolutionary process.

On panels $A$ and $B$ the geographical areas where the virus strains were detected frequently are indicated above the peaks of genetic diversity.

On panel C, plots estimated for the EV-71 subgenogroups $C_{1}$ and $C_{2}$ are superimposed on the same time scale. The epidemic peaks are numbered. The sporadic introductions of EV-71/C4 strains in European Union Member States (as described in this study and [34]) and Canada are indicated with open triangles. The introduction events in Russia are shown with full triangles. The introduction events of the EV-71 subgenogroup $B_{5}$ in Denmark [30] and France [31] are shown with diamonds.

are mainly restricted to Asian countries while subgenogroups $C_{1}$ and $C_{2}$ are chiefly found in Europe [20]. Many aspects of the epidemiological and evolutionary dynamics of circulating virus strains remain unknown. In particular, how virus transmission occurs over time, across space, and among genogroups has not been extensively studied in geographical areas other than eastern and south-east Asia [21]. We investigated the evolutionary dynamics of EV-71 to determine the origin of virus strains sampled in Europe and their relationships with viruses reported elsewhere in the world. To this aim, we used large sequence datasets of virus isolates sampled worldwide since the mid-1980s, which were mainly representative of EV-71 subgenogroups circulating in Europe and eastern and southeast Asia. We estimated the genetic diversity with the $1 \mathrm{D}$ gene encoding the capsid viral protein 1 (hereafter designated $1 \mathrm{D}^{\mathrm{VP} 1}$ ) over time, across geographical areas, and among subgenogroups, something that, to our knowledge, has never been done on such a large scale before. 


\section{Methods}

Virus samples, gene amplification, and nucleotide sequencing

The enterovirus samples studied consisted of 64 EV-71 strains isolated in Austria $(n=1)$, France $(n=43)$, Germany $(n=12)$, and Hungary $(n=8)$ between 2003 and 2013, and 33 strains recovered between 2001 and 2013 in Azerbaijan $(n=1)$, Kazakhstan $(n=1)$, Kyrgyzstan $(n=1)$, Ukraine $(n=1)$ and Russia $(n=29)$. The EV-71 isolates were collected as part of routine clinical work-up.

The $1 D^{\mathrm{VP} 1}$ sequences were determined in the virus isolates with the previously described genotyping methods $[12,22]$. The complete $1 \mathrm{D}$ gene sequence was amplified with either standard single-round (amplicon length of $1,200 \mathrm{bp}$ ) or semi-nested (amplicon of 2,240 bp) polymerase chain reaction (PCR) assays. The nucleotide (nt) sequences determined in this study were deposited in publicly available sequence databases under the accession numbers HG934162 to HG934296.

\section{Data collection and compilation of nucleotide} sequence datasets

The sequences determined were analysed with sequences obtained from GenBank. The $1 \mathrm{D}^{\mathrm{VP} 1}$ sequence datasets were constructed by collating all GenBank entries (as of July 2013 for the $1 D^{\mathrm{VP}_{1}}$ locus) including a sequence of the VP1 capsid protein for any human isolate of EV-71. Entries reporting nt sequences of $<891 \mathrm{nt}$ were discarded and only sequences with fully specified dates (year) and countries of origin were used. The sequence datasets were constructed with BioEdit v.7.2.5 software (http://www.mbio.ncsu.edu/bioedit/ bioedit.html) and were compiled with all sequences determined in our laboratory. The EV- $711 D^{\mathrm{VP}_{1}}$ gene sequences were distributed into five datasets corresponding to subgenogroups $B_{4} / B_{5}(n=217$ sequences), $C_{1}(n=280), C_{2}(n=322)$, and $C_{4}(n=675)$. On the basis of earlier phylogenetic data indicating that subgenogroups $\mathrm{B}_{4}$ and $\mathrm{B}_{5}$ had a common ancestor [21], we analysed jointly their sequences to increase the sample size. To investigate the $\mathrm{EV}-71 / \mathrm{C}_{4}$ subgenogroup, an initial dataset of 775 complete sequences was downsized to 675 by removing all sequences but one in genetic clusters containing multiple sequences with $\geq 99.5 \% \mathrm{nt}$ identity with one another.

\section{Coalescent estimation of divergence dates and evolutionary rates}

Genealogical trees of EV-71 subgenogroups were reconstructed with the Bayesian evolutionary analysis by sampling trees (BEAST) v1.7.5 programme [23]. Uncorrelated lognormal prior distributions of substitution rates among lineages were used for the molecular clock model [24]. The evolutionary history was reconstructed with the substitution general time reversible (GTR) model. The phylogenetic parameters were coestimated in the different analyses by a Markov chain Monte Carlo (MCMC) process involving $100 \times 10^{6}$ to 200 $x 10^{6}$ generations to ensure convergence of parameter estimates. The Tracer v.1.5 programme (http://evolve. zoo.ox.ac.uk/Evolve/Software.html) was used to check for convergence and mixing (operator effective sample size $>200$ ). The trees estimated with the MCMC procedure were sampled to obtain a final 20,000 trees. Mean estimates and $95 \%$ highest probability density (HPD) intervals calculated for each operator were compiled by analysing the output files obtained from the BEAST programme with the Tracer v.1.5 programme. Maximum clade credibility (MCC) trees were calculated with the TreeAnnotator V.1.7.5 programme (http://evolve.zoo. ox.ac.uk/Evolve/Software.html) and topological support was assessed by estimating the values of the posterior probability (pp) density of each node.

\section{Reconstruction of the demographic history and geographical spread of enterovirus subgenogroups}

The Bayesian skyline plot model (BSP) does not assume a pre-defined model of demography [25]. It infers a demographic parameter representing 'virus population size', also referred to as relative genetic diversity, using the coalescent theory and temporal information of the molecular data (virus collection date). Genetic diversity is the product of the effective size of the virus population $\left(\mathrm{N}_{\mathrm{e}}\right)$ and the generation time $(\mathrm{t})$, and reflects the effective number of infections averaged over time under the assumption of a neutral evolutionary process. In our study, this model was used to investigate possible variations in virus population size over time for subgenogroups $B_{4}, B_{5}, C_{1}, C_{2}$, and $C_{4}$ to describe past epidemiological events on a continuous time scale using large sequence datasets for the $1 \mathrm{D}^{\mathrm{VP} 1}$ gene. EV-71 infections are characterised by epidemic transmission in a number of countries, a high rate of asymptomatic or pauci-symptomatic infections, and short-lived acute infections in susceptible individuals. Accordingly, the skyline plot model appears epidemiologically better suited than the other demographic models (constant population size, exponential growth, logistic growth, and expansion growth) for modelling the spread of EV-71 populations and thus for reconstructing the diffusion of HFMD over time and across geographical regions. The BSP analyses were done with a lognormal distribution (piecewise-constant population size model) to account for variation in substitution rates among the phylogenies. The countries where the virus strains were collected were used as discrete character states (or traits) in the phylogeographical analysis to estimate changes of geographical locations on the phylogenetic trees [26]. The geographical locations of the ancestral nodes were co-estimated with the phylogeny by using a discrete phylogeographical diffusion model (symmetrical substitution model). Bayesian stochastic search variable selection was also used for identifying the statistically significant transition rates between locations. TreeAnnotator software was used to produce the MCC tree, with the branches coloured by traits. 


\section{FIGURE 3}

Spatial and temporal distribution of enterovirus 71 lineages showing the geographical dissemination of subgenogroups $\mathrm{C} 1$ (panel A) and C2 (panel B)

A.

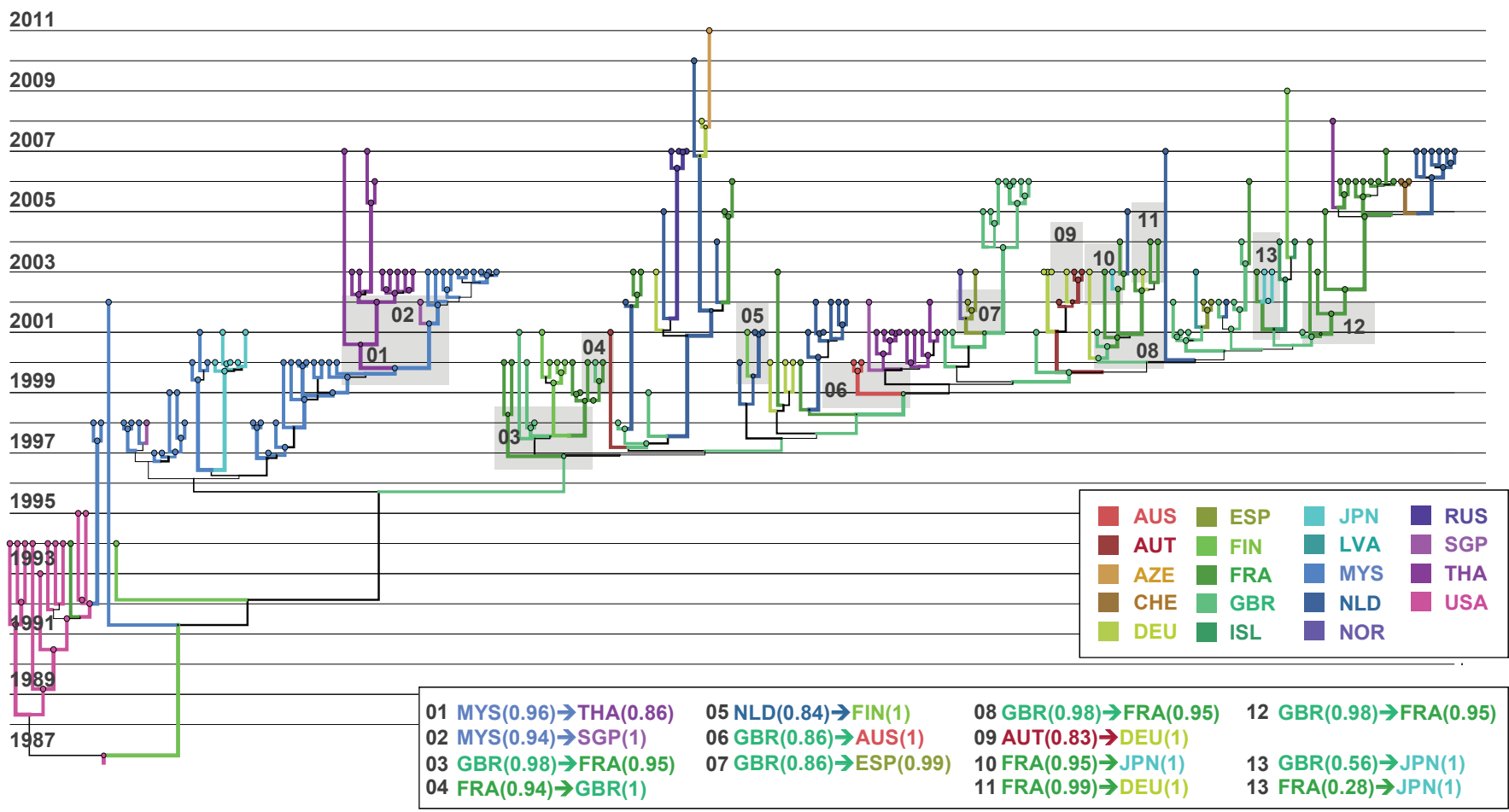

B.

$\underline{2013}$

$\underline{2011}$

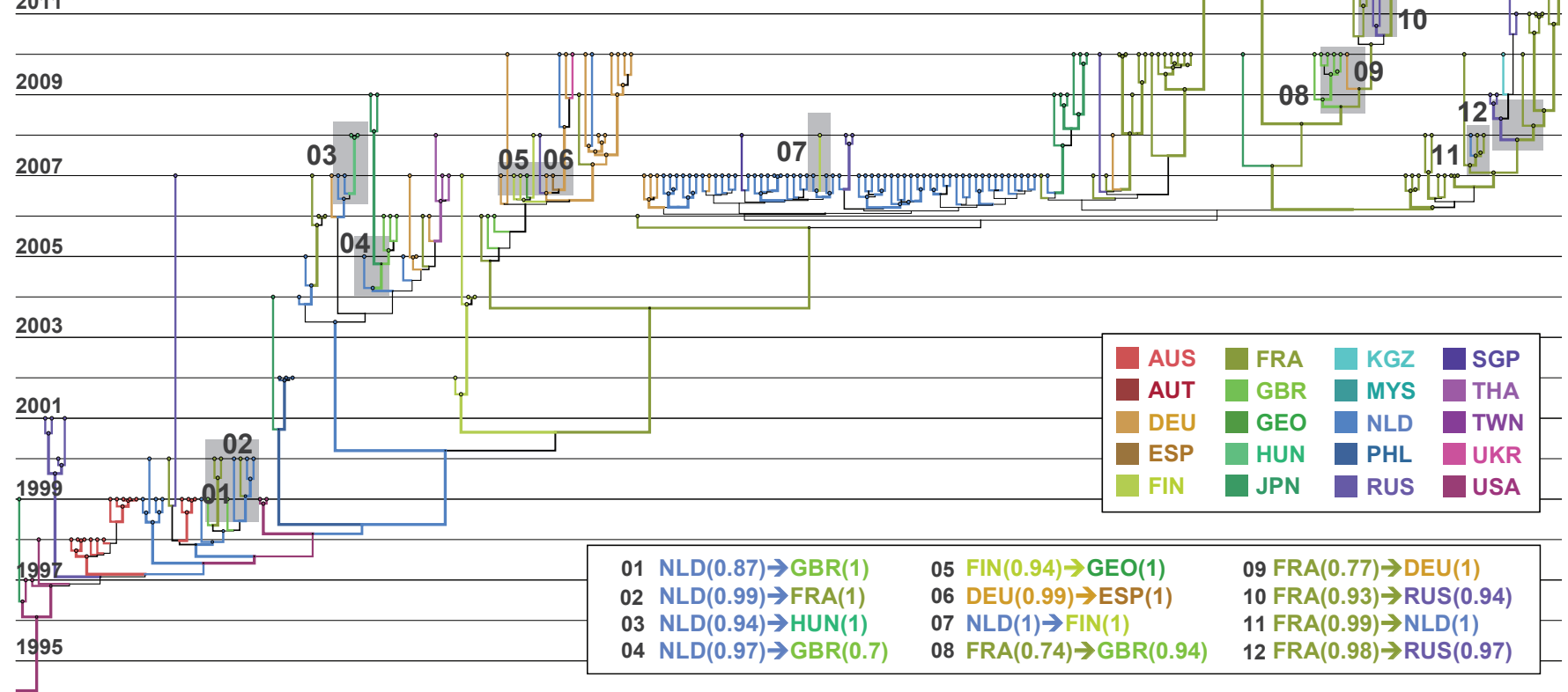

AUS: Australia; AUT: Austria; AZE: Azerbaijan; CHE: Switzerland; DEU: Germany; ESP: Spain; FIN: Finland; FRA: France; GBR: United Kingdom; GEO: Georgia; HUN: Hungary; ISL: Iceland; JPN: Japan; KGZ: Kyrgyzstan; LVA: Latvia; MYS: Malaysia; NLD: Netherlands; NOR: Norway; PHL: Philippines; RUS: Russia; SGP: Singapore; THA: Thailand; TWN: Taiwan; UKR: Ukraine; USA: United States.

The MCC tree was obtained from a Bayesian MCMC analysis (discrete phylogeographical model) and shows for more clarity the most relevant parts of tree topologies (time is indicated as calendar years). The FigTree programme was used to display a number of information. Branches and circles at the tree nodes are coloured according to the geographical location that had the highest probability. The size of each circle represents the location probability. Line width indicates the posterior probability of the corresponding lineage (thick lines indicate high posterior probability values). The tree topologies were used to determine the phylogenetic patterns (numbered sequentially) showing the most probable virus spread events. The main features of the most probable virus spread events (i.e. geographical location, location probability, and spread direction) are indicated in the figure. 
Geographical spread of enterovirus 71 subgenogroups C1 (green) and C2 (blue) among different countries, 1998-2011

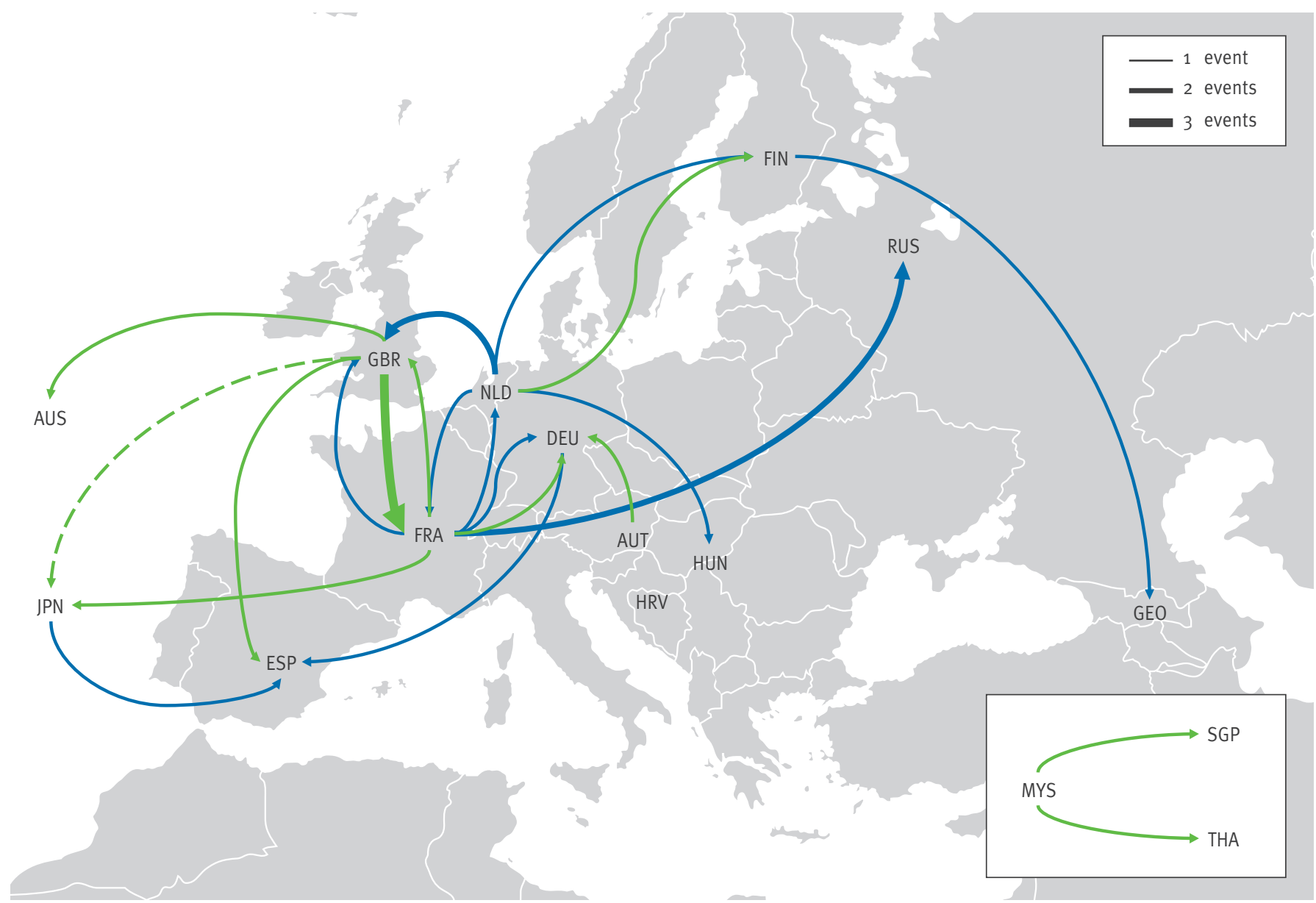

AUS: Australia; AUT: Austria; DEU: Germany; ESP: Spain; FIN: Finland; FRA: France; GBR: United Kingdom; GEO: Georgia; HRV: Croatia; HUN: Hungary; JPN: Japan; MYS: Malaysia; NLD: Netherlands; RUS: Russia; SGP: Singapore; THA: Thailand.

The most probable virus spread events of EV-71 strains analysed in Figure 3 are schematically represented in the Figure above. Line width represents the number of spread events inferred between two countries as indicated in the upper right scale.

\section{Results}

Phylogenies of five enterovirus 71 subgenogroups estimated with datasets of the $1 D$ gene encoding the capsid viral protein 1 We used the large number of $1 \mathrm{D}^{\mathrm{VP} 1}$ sequences determined in circulating strains to obtain a comprehensive comparison of virus transmission among EV-71 subgenogroups. The country of virus isolation was checked for each sequence to determine the distribution in four main geographical areas: (i) eastern and south-east Asia as well as Australia, (ii) Azerbaijan, Georgia, Kazakhstan, Kyrgyzstan, Russia and Ukraine (iii) Europe (whereby data originated from EU Member States, as well as Iceland, Norway and Switzerland) and (iv) North America, as observed in the genealogies. The phylogenies displayed a 'ladder-like' shape mainly characterised by a long trunk with short side branches, evidence of rapid extinction of lineages over time (Figure 1). These topologies arose from a combined effect of temporal sampling in various countries and rapid coalescence. A number of long side branches were also inferred in the $\mathrm{C}_{1}, \mathrm{C}_{2}$, and $\mathrm{C}_{4}$ trees, which indicated transmission of lineages over time and the persistence of these lineages alongside more recent ones. Of the subgenogroups examined, all but one (i.e. subgenogroup (1) were inferred to arise in the 1990 s (Figures $1 \mathrm{~A}, 1 \mathrm{C}, 1 \mathrm{D})$. The time to most recent common ancestor (TMRCA) of subgenogroup EV-71/C1 was estimated in 1983.5 (95\% HPD: 1982.2-1984.4) (Figure 1B). Most $\mathrm{B}_{4}, \mathrm{~B}_{5}$ and $\mathrm{C}_{4}$ lineages were restricted to eastern and south-east Asia while subgenogroups $C_{1}$ and C2 originated from this geographical area as well as European countries and Russia over the sampling periods. After 2000, several phylogenetically independent EV-71 C2 lineages were inferred to have circulated in Europe, during three main periods in 2007, 2010, and 2013. The $C_{4}$ phylogeny also included a few lineages in EU Member States and Russia, which suggests that a number of persons in these countries sporadically acquired the virus from individuals elsewhere in the world, most probably eastern and south-east Asia. 


\section{FIGURE 5}

Bayesian analyses of the spatial and temporal spread of an enterovirus 71 subgenogroup C4 lineage, 2002-2006

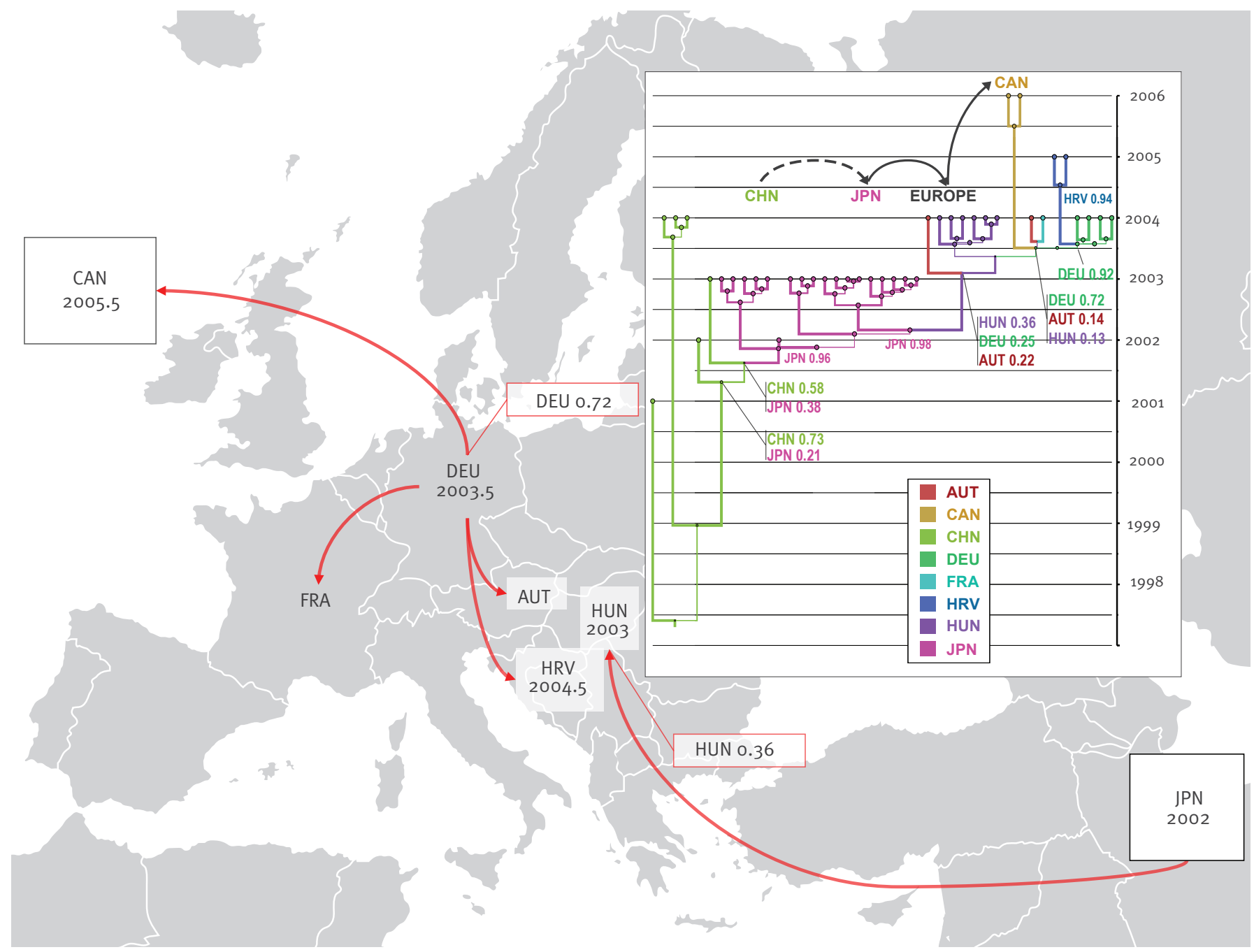

AUT: Austria; CAN: Canada; CHN: China; DEU: Germany; FRA: France; HRV: Croatia; HUN: Hungary; JPN: Japan.

The topology was drawn with the sequence sample corresponding to the subtree indicated in Figure 1 . The geographical locations and the location probabilities estimated with a discrete phylogeographical model are indicated for the most relevant nodes. The most probable virus spread events are shown in the map insert.

Genealogy-based population dynamics of enterovirus 71 subgenogroups

The demographic histories showed variations between the five subgenogroups. The pattern estimated by our coalescent-based reconstruction for subgenogroup $\mathrm{C}_{4}$ clearly indicated an annual series of increases in genetic diversity interspersed with genetic bottlenecks. This pattern was caused by the large HFMD epidemics recently observed in China [3] and confirmed the usefulness of the BSP model for investigating EV-71 transmission.

We provide the detailed data obtained for the most frequent subgenogroups ( $\mathrm{C}_{1}$ and $\mathrm{C}_{2}$ ) in Europe (Figure 2). The pattern estimated for subgenogroup $\mathrm{C}_{1}$ (Figure 2A) depicted successive variations in virus population size. This demographic pattern was consistent with a few outbreaks over time and across different geographical areas as documented by epidemiological data $[20,27]$. This pattern may also indicate uninterrupted global circulation of the virus.

The pattern reconstructed for $\mathrm{EV}-71 / \mathrm{C}_{2}$ disclosed two distinct rises in virus population size (Figure 2B). The subgenogroup experienced a first sharp increase in 1998 that coincided with the occurrence of a large HFMD epidemic in Taiwan [28]. The virus population size then slowly decreased over several years. The second exponential rise in virus population size (in 2007) was of a high magnitude. This major increase in virus genetic diversity coincided with the occurrence of an outbreak in the Netherlands [29] and a concomitant rise in virus circulation in Austria, France, and Germany [12]. The transmission of the virus remained at a sustained high level after the 2007 epidemic, yet the different lineages sampled in 2010 and 2013 (Figure 1C) 
were not associated with increases in virus population size.

A combination of the $C_{1}$ and $C_{2}$ patterns showed that the decrease in the EV-71/C1 population size in 1996 was concurrent with the initial occurrence of EV-71/ C2 (Figure 2C). The 1998 increase in virus population size (Taiwan epidemic, subgenogroup $\mathrm{C}_{2}$, peak 1 ) coincided with a lower genetic diversity of EV-71/C 1 . The marked increase in $C_{2}$ genetic diversity in 2007 (peak 5) also occurred in Europe during a period of low $C_{1}$ diversity. The $C_{1}$ pattern showed three yearly ascending rises (peaks 2, 3, and 4) during a long period of low genetic diversity of the $\mathrm{C}_{2}$ virus. The population size of both $C_{1}$ and $C_{2}$ subgenogroups remained low over the years 2004 to 2006 and 2008 to 2013 , periods during which the EV-71/C4 subgenogroup occurred in several European countries (Austria, Croatia, France, Germany, and Hungary) and Canada (see below). EV-71/B5 caused an outbreak in Denmark (2007) [30] and a sporadic infection in France (2013) [31].

\section{Spread of enterovirus 71/C1 between distinct countries}

Spread of EV-71 between different countries was investigated with the MCC trees of the above analyses. A phylogenetic pattern indicative of a probable virus spread event between two nodes was defined as follows: (i) the nodes exhibited pp>0.9, (ii) the inferred location probabilities were $>0.7$ at both nodes, and (iii) the difference between the TMRCA values or the $95 \%$ HPD interval values of nodes were in a range of one year. With this conservative approach, the MCC tree topology inferred for subgenogroup $\mathrm{C}_{1}$ indicated a total of 13 consistent virus transmission events (Figure $3 A$ ). Eight phylogenetic patterns (numbered 03-05, 07-09, 11 and 12 in Figure $3 A$ ) indicated that the virus was transmitted between persons within various European countries. The United Kingdom, with a probability of 0.98 , was likely the country of origin in three inferred virus spread events (numbered 03, 08, and 12, Figure $3 A$ ) to France (probabilities of 0.95 ) and one to Spain (0.99). France was inferred as the origin of two spread events, respectively to Germany and the United Kingdom. The EV-71/C 1 tree also showed that virus spread occurred from Austria (location probability, 0.83 ) to Germany and from the Netherlands (0.84) to Finland. Three other phylogenetic patterns (numbered 06,10 , and 13 in Figure $3 A$ ) were indicative of long distance virus spread from a EU Member State to either Australia or Japan ( $n=2$ events). In the spread event number 13 (Figure $3 \mathrm{~A}$ ), the probability of the United Kingdom being the source country was estimated to be 0.56 against 0.28 for France. Two consistent phylogenetic patterns showed virus spread from Malaysia (location probability $\geq 0.94$ ) respectively to Singapore and to Thailand.

A schematic representation of all virus spread events described above is shown in Figure 4.
Spread of enterovirus 71/C2 between distinct countries

In the MCC tree inferred for subgenogroup C2 (Figure 3B), 12 phylogenetic patterns indicated that the virus spread between distinct European countries, Georgia, and Russia. The most frequent likely countries of origin of infected persons in the inferred virus spread events were the Netherlands ( $n=5$ events; location probability range: $0.87-1)$ and France $(n=5 ; 0.74-0.98)$ followed by Finland and Germany. The 2007 epidemic lineage in the Netherlands was involved in only one virus spread event (Figure 3; number 7). Three virus spread events were estimated between distant countries: from Finland to Georgia and from France to Russia. An additional event of spread of the virus from Japan (location probability: 0.85) to Spain was also inferred in the genealogy before 1995 (not shown in Figure 3B). All the virus spread events occurring after 1998 are shown in Figure 4 .

\section{Spread of enterovirus 71/C4 between distinct countries}

We analysed separately a sequence subset corresponding to the lineage highlighted in Figure 1D. The distributions of geographical location probabilities were indicated for the most relevant nodes in the MCC tree inferred for this sequence subset and were used to investigate virus spread between countries (Figure 5). The phylogeny pattern indicated that between 2001 and 2002 a C4 virus strain spread from China to Japan, and from there to the EU in early 2003. The virus was disseminated in different EU Member States between 2003 and 2004. It was sampled in Croatia in 2005 and the phylogenetic analysis estimated a probability of 0.92 that the source country was Germany. The same virus strain also moved from the EU to Canada (sampling year 2006). The phylogenetic analysis suggested that the virus was spread from Germany with a probability of 0.72 .

\section{Discussion}

The recent reporting of sporadic circulation of EV-71 strains of the 'Asian subgenogroups' B5 [30,31] and $\mathrm{C}_{4}$ [32-34] in European countries makes it important to determine the evolutionary origins and spatiotemporal spread of EV-71 infections in geographical areas other than eastern and south-east Asia. There is a large body of literature on the molecular epidemiology of EV-71 [35] but, to our knowledge, the spread of this virus has not yet been analysed explicitly with recently developed phylogeographical models [26]. In this report, we therefore applied demographic and phylogeographical analyses to compare the population structure and the dissemination patterns of EV-71 subgenogroups in Europe and Asia. We found that the EV-71 subgenogroups exhibit distinct phylodynamic patterns and that these patterns differed from those of past epidemics in frequency and geographical location. The transmission history of subgenogroup C 2 was characterised by fewer increases in genetic diversity than in subgenogroups $\mathrm{C}_{1}$ and $\mathrm{C}_{4}$. For instance, the genetic diversity pattern 
of subgenogroup C2 shows clearly the two outbreaks (Taiwan, 1998 and the Netherlands, 2007) documented in the literature $[28,29]$. The variations in genetic diversity over the study period also reflect gaps in virus sampling over time and surveillance differences between countries. In Europe, EV-71 sequences are determined solely in patients admitted to hospitals and in a few countries only, whereas in a number of Asian countries real-time epidemiological data are obtained from sentinel surveillance of HFMD cases. In this study, we used only full-length EV-71 $1 \mathrm{D}^{\mathrm{VP} 1}$ sequences because phylogenetic trees based on short-length sequences did not provide a high level of statistical confidence. Partial nt sequences were notably determined for virus genotyping and ranged over different parts of the $1 D^{V^{P_{1}}}$ gene. Accordingly, we had to discard a certain amount of sequence data, some from Europe, which might have been helpful in phylogenetic analysis of virus spread.

We show how transmission of EV-71 strains (subgenogroups $\mathrm{C}_{1}, \mathrm{C}_{2}$, and $\mathrm{C}_{4}$ ) occurs in Europe as discrete and temporally defined virus introductions, occasionally followed by limited local spread. The only exception to this pattern was the epidemic expansion in the Netherlands in 2007. Interestingly, we found limited phylogenetic evidence that the Dutch outbreak was the source of a large spread to other European countries. The phylogenetic patterns also show that European countries may experience multiple virus introduction events within the same year. This dissemination mode was observed within particular countries (e.g. France, Germany) for both subgenogroups $\mathrm{C}_{1}$ and $\mathrm{C}_{2}$ but was more clearly seen during three waves of infections in 2007, 2010, and 2013 (subgenogroup C2). The epidemiological and biological factors involved are unknown but the occurrence of these infection waves is consistent with the hypothesis that the sustained circulation of an EV-71 strain throughout Europe depends on the proportion of susceptible hosts in different countries. The data may alternatively indicate that the immunity elicited by the $C_{1}$ and $C_{2}$ infections is cross-protective, as suggested by earlier studies that identified common epitopes and suggested that human immune sera among virus strains of different EV-71 subgenogroups have cross-neutralization properties [36]. In this respect, we also provide phylogenetic evidence that the spread of the $\mathrm{C} 2$ virus across European countries in 2007 happened at a time when $C_{1}$ infections had been transmitted at low rates for at least three years.

It is particularly noteworthy that the transmission of EV-71 strains $C_{1}$ and $C_{2}$ in Europe is mainly dependent on the frequency of virus spread events between neighbouring countries. This has been previously described for EV-71 [37] and for another EV, coxsackievirus $\mathrm{B}_{5}$ [38]. The present study also indicates that the long-term survival of EV-71 ( $\mathrm{C}_{1}$ and $\mathrm{C}_{2}$ ) depends on continued virus transmission between individuals across larger geographical areas, notably Russia and Asia. We also reconstructed a consistent transmission chain caused by a $\mathrm{C}_{4}$ virus strain throughout Europe
(Austria, Croatia, France, Germany, and Hungary), which suggests that the virus persisted between 2003 and 2005 . The chain was brought about by a virus from Japan which eventually reached Canada between 2005 and 2006. Transmission of the $C_{1}$ and $C_{2}$ infections was low during the whole period in Europe. Similarly, the recent $\mathrm{C}_{4}$ infections in France (2012), and Russia (2013) arose during a transmission trough of both $C_{1}$ and $\mathrm{C}_{2}$ infections. Thus, transcontinental transmission events between individuals should be considered in the global epidemiology of the virus: they provide the epidemiological bases for explaining the long-term survival of lineages despite abrupt extinctions that drastically reduce virus diversity after the occurrence of outbreaks in particular locations.

Earlier hypotheses proposed that yearly epidemics of EV-71 in Japan arose from spread of the virus from neighbouring countries [37]. We suggest that virus strains are sustained by complex migration dynamics involving Europe and Russia, and possibly other geographical regions. This argument is lent weight by a recent study reporting the occurrence of EV-71 $\mathrm{C}_{4}$ in Denmark in 2012 and 2013 [39]. Our Bayesian analysis of the partial $1 D^{V P_{1}}$ sequences showed that the $C_{4}$ viruses isolated in Denmark were distributed in two distinct phylogenetic clusters, which suggests independent virus sources (data not shown). Thus, the frequency of epidemics in Asian countries, the involvement of EV-71 in neurological conditions, the recent isolation of $\mathrm{C}_{4}$ variant strains in Europe, and the spread patterns of the virus between distant and neighbouring countries underline a need for enhanced surveillance of EV-71 infections in Europe using common enterovirus genotyping methods.

\section{Acknowledgements}

The authors are grateful to the following people involved in the French Network of Enterovirus Surveillance, $\mathrm{Dr}$ D. Hecquet and Prof. G. Duverlie (Amiens); Dr A. Paquin, Dr A. Ducancelle, and Prof. F. Lunel-Fabiani (Angers); Dr MC. Legrand and Prof. Christopher Payan (Brest); Dr J. Petitjean and Prof. Astrid Vabret (Caen); Dr Christine Morel-Baccard and Prof. Patrice Morand (Grenoble); Prof. E. Schvoerer (Nancy); Dr M. Coste-Burel (Nantes); Dr A. Bourgoin and Prof. G. Agius (Poitiers); Dr G. Lagathu and Prof. R. Colimon (Rennes); Dr Jean-Michel Mansuy and Prof. Jacques Izopet (Toulouse); Dr Stéphanie Marque-Juillet (Versailles), for providing us with virus samples used in this study. The authors are also grateful to the following people for providing us with virus samples collected in other European countries, Dr A. Marchut and Dr M. Kozmane Török (National Center for Epidemiology, Budapest, Hungary). The authors are indebted to Prof. Gisela Enders, director of Laboratory Prof. Gisela Enders \& Kollegen MVZ (Stuttgart, Germany) for help and constant involvement in our European molecular epidemiology studies. We acknowledge the technical contribution of Gwendoline Jugie, Nathalie Rodde, and Isabelle Simon for helpful assistance with virus genotyping. We thank Jeffrey Watts for help with preparing the English manuscript. 
Conflict of interest

None declared.

\section{Authors' contributions}

Chervin Hassel: performed sequencing and phylogenetic analyses, provided and analysed the data, and provided comments on the manuscript; Audrey Mirand: did isolation and characterisation of virus strains in France, performed sequencing, and analysed the data, and provided comments on the manuscript; Alexander Lukashev: did isolation and characterisation of virus strains, provided sequence data before publication, and provided comments on the manuscript; Elena Terletskaia-Ladwig: did isolation and characterisation of virus strains in Germany, provided clinical data, and provided comments on the manuscript; Agnes Farkas: did isolation and characterisation of virus strains in Hungary, and provided clinical data; Isabelle Schuffenecker: did isolation and characterisation of virus strains in France, provided clinical data; Sabine Diedrich: did isolation and characterisation of virus strains in Germany, provided clinical data; Hartwig P Huemer: did isolation and characterisation of virus strains in Austria, provided clinical data; Christine Archimbaud: did isolation and characterisation of virus strains in France; Hélène Peigue-Lafeuille: designed the protocol of investigation, provided comments on the manuscript; Cécile Henquell: designed the protocol of investigation, analysed the data, provided comments on the manuscript; Jean-Luc Bailly: designed the protocol of investigation, set up the field epidemiology and contacted all investigators, analysed the data, and wrote the first draft.

\section{References}

1. OoiMH, WongSC, LewthwaiteP, CardosaMJ, SolomonT. Clinical features, diagnosis, and management of enterovirus 71.Lancet Neurol. 2010;9(11):1097-105. DOI: 10.1016/S14744422(10)70209-X PMID: 20965438

2. Taiwan Enterovirus Epidemic Working Group,HoM, ChenER, HsuKH, TwuSJ, ChenKT, TsaiSF, et al. . An epidemic of enterovirus 71 infection in Taiwan.N Engl J Med. 1999;341(13):929-35. DOI: 10.1056/NEJM199909233411301 PMID: 10498487

3. XingW, LiaoQ, ViboudC, ZhangJ, SunJ, WuJT, et al. Hand, foot, and mouth disease in China, 2008-12: an epidemiological study. Lancet Infect Dis. 2014;14(4):308-18. DOI: 10.1016/ S1473-3099(13)70342-6 PMID: 24485991

4. ChangLY, KingCC, HsuKH, NingHC, TsaoKC, LiCC, et al. Risk factors of enterovirus 71 infection and associated hand, foot, and mouth disease/herpangina in children during an epidemic in Taiwan. Pediatrics. 2002;109(6):e88. DOI: 10.1542/ peds.109.6.e88 PMID: 12042582

5. Lee MS, Tseng FC, Wang JR, Chi CY, Chong P, Su IJ. Challenges to licensure of enterovirus 71 vaccines. PLoS Negl Trop Dis. 2012;6(8): e1737.

6. Blomberg,, LyckeE, AhlforsK, JohnssonT, WolontisS, von ZeipelG. Letter: New enterovirus type associated with epidemic of aseptic meningitis and-or hand, foot, and mouth disease.Lancet. 1974;304(7872):112. DOI: 10.1016/S01406736(74)91684-5 PMID: 4136956

7. ChumakovM, VoroshilovaM, ShindarovL, Lavroval, GrachevaL, KorolevaG, et al. Enterovirus 71 isolated from cases of epidemic poliomyelitis-like disease in Bulgaria. Arch Virol. 1979;60(3-4):329-40. DOI: 10.1007/BF01317504 PMID: 228639

8. ShindarovLM, ChumakovMP, VoroshilovaMK, BojinovS, VasilenkoSM, Iordanovl, et al. Epidemiological, clinical, and pathomorphological characteristics of epidemic poliomyelitislike disease caused by enterovirus 71. J Hyg Epidemiol Microbiol Immunol. 1979;23(3):284-95.PMID: 231067

9. SohierR. Enterovirus 71 surveillance: France.Wkly Epidemiol Rec.1979;54(28):219.

10. DiedrichS, WeinbrechtA, SchreierE. Seroprevalence and molecular epidemiology of enterovirus 71 in Germany. Arch Virol. 2009;154(7):1139-42. DOI: 10.1007/s00705-009-0413-x PMID: 19506798

11. RabenauHF, RichterM, DoerrHW. Hand, foot and mouth disease: seroprevalence of Coxsackie A16 and Enterovirus 71 in Germany. Med Microbiol Immunol (Berl). 2010;199(1):45-51. DOI: 10.1007/s00430-009-0133-6 PMID: 19941005

12. MirandA, Schuffeneckerl, HenquellC, BillaudG, JugieG, FalconD, et al. Phylogenetic evidence for a recent spread of two populations of human enterovirus 71 in European countries. J Gen Virol. 2010;91(Pt 9):2263-77. DOI: 10.1099/ vir.0.021741-0 PMID: 20505012

13. RothB, EndersM, ArentsA, PfitznerA, Terletskaia-LadwigE. Epidemiologic aspects and laboratory features of enterovirus infections in Western Germany, 2000-2005.J Med Virol. 2007;79(7):956-62. DOI: 10.1002/jmv.20917 PMID: 17516529

14. Dutch Working Group for Clinical Virology,van der SandenS, KoopmansM, UsluG, van der AvoortH. Epidemiology of enterovirus 71 in the Netherlands, 1963 to 2008.J Clin Microbiol. 2009;47(9):2826-33. DOI: 10.1128/JCM.00507-09 PMID: 19625480

15. ValletS, Legrand QuillienMC, DaillandT, PodeurG, GouriouS, Schuffeneckerl, et al. Fatal case of enterovirus 71 infection, France, 2007. Emerg Infect Dis. 2009;15(11):1837-40. DOI: 10.3201/eid1511.090493 PMID: 19891879

16. KassabS, SaghiT, BoyerA, LafonME, GrusonD, LinaB, et al. Fatal case of enterovirus 71 infection and rituximab therapy, france, 2012. Emerg Infect Dis. 2013;19(8):1345-7. DOI: 10.3201/ eid1908.130202 PMID: 23880543

17. Schuffeneckerl, MirandA, AntonaD, HenquellC, ChomelJJ, ArchimbaudC, et al. Epidemiology of human enterovirus 71 infections in France, 2000-2009. J Clin Virol. 2011;50(1):50-6. DOI: 10.1016/j.jcv.2010.09.019 PMID: 21035387

18. HonkanenH, OikarinenS, PakkanenO, RuokorantaT, PulkkiMM Laitinen $\mathrm{OH}$, et al. Human enterovirus 71 strains in the background population and in hospital patients in Finland. Clin Virol. 2013;56(4):348-53. DOI: $10.1016 / j$.jcv.2012.11.018 PMID: 23261080

19. BessaudM, RazafindratsimandresyR, NougairèdeA, Joffret $M L$, DeshpandeJM, Dubot-PérèsA, et al. Molecular comparison and evolutionary analyses of VP1 nucleotide sequences of new African human enterovirus 71 isolates reveal a wide genetic diversity. PLoS ONE. 2014;9(3):e90624. DOI: 10.1371/journal. pone.0090624 PMID: 24598878

20. McMinnPC. Recent advances in the molecular epidemiology and control of human enterovirus 71 infection.Curr Opin Virol. 2012;2(2):199-205. DOI: 10.1016/j.coviro.2012.02.009 PMID: 22482716

21. TeeKK, LamTT, ChanYF, BibleJM, KamarulzamanA, TongCY, et al. Evolutionary genetics of human enterovirus 71: origin, population dynamics, natural selection, and seasonal periodicity of the VP1 gene. J Virol. 2010;84(7):3339-50. DOI: 10.1128/JVI.01019-09 PMID: 20089660

22. MirandA, HenquellC, ArchimbaudC, UghettoS, AntonaD, BaillyJL, et al. Outbreak of hand, foot and mouth disease herpangina associated with coxsackievirus A6 and A10 infections in 2010, France: a large citywide, prospective observational study. Clin Microbiol Infect. 2012;18(5):E110-8. DOI: 10.1111/j.1469-0691.2012.03789.x PMID: 22404077

23. DrummondAJ, SuchardMA, XieD, RambautA. Bayesian phylogenetics with BEAUti and the BEAST 1.7. Mol Biol Evol. 2012;29(8):1969-73. DOI: 10.1093/molbev/mss075 PMID: 22367748

24. DrummondAJ, HoSY, PhillipsMI, RambautA. Relaxed phylogenetics and dating with confidence.PLoS Biol. 2006;4(5):e88. DOI: 10.1371/journal.pbio.0040088 PMID: 16683862

25. DrummondAJ, RambautA, ShapiroB, PybusOG. Bayesian coalescent inference of past population dynamics from molecular sequences. Mol Biol Evol. 2005;22(5):1185-92. DOI: 10.1093/molbev/msi103 PMID: 15703244

26. LemeyP, RambautA, DrummondAJ, SuchardMA. Bayesian phylogeography finds its roots.PLOS Comput Biol. 2009;5(9):e1000520. DOI: 10.1371/journal.pcbi.1000520 PMID: 19779555

27. YipCC, LauSK, WooPC, YuenKY. Human enterovirus 71 epidemics: what's next?Emerg Health Threats J. 2013;6(0):19780. DOI: 10.3402/ehtj.v6io.19780 PMID: 24119538

28. WangJR, TuanYC, TsaiHP, YanJJ, LiuCC, SulJ. Change of major genotype of enterovirus 71 in outbreaks of hand-foot-andmouth disease in Taiwan between 1998 and 2000.J Clin Microbiol. 2002;40(1):10-5. DOI: 10.1128/JCM.40.1.10-15.2002 PMID: 11773085

29. van der SandenS, van der AvoortH, LemeyP, UsluG, KoopmansM. Evolutionary trajectory of the VP1 gene of human enterovirus 71 genogroup $B$ and C viruses.J Gen Virol. 2010;91(Pt 8):1949-58. DOI: 10.1099/vir.0.019695-o PMID: 20375223

30. BadranSA, MidgleyS, AndersenP, BöttigerB. Clinical and virological features of enterovirus 71 infections in Denmark, 
2005 to 2008 .Scand J Infect Dis. 2011;43(8):642-8. DOI: 10.3109/00365548.2011.577094 PMID: 21526904

31. MirandA, MoletL, HasselC, Peigue-LafeuilleHC, RozenbergF, BaillyJL, et al. Enterovirus A71 subgenotype B5, France, 2013. Emerg Infect Dis. 2015;21(4):707-9.DOI: 10.3201/ eid2104.141093 PMID: 25811300

32. OrtnerB, HuangCW, SchmidD, Mutzl, WewalkaG, AllerbergerF, et al. Epidemiology of enterovirus types causing neurological disease in Austria 1999-2007: detection of clusters of echovirus 30 and enterovirus 71 and analysis of prevalent genotypes. J Med Virol. 2009;81(2):317-24. DOI: 10.1002/ jmv.21374 PMID: 19107980

33. KapusinszkyB, SzomorKN, FarkasA, TakácsM, BerencsiG. Detection of non-polio enteroviruses in Hungary 2000-2008 and molecular epidemiology of enterovirus 71, coxsackievirus A16, and echovirus 30.Virus Genes. 2010;40(2):163-73. DOI: 10.1007/S11262-009-0440-4 PMID: 20044791

34. Schuffeneckerl, HenquellC, MirandA, Coste-BurelM, MarqueJuilletS, DesboisD, et al. New introductions of enterovirus 71 subgenogroup $\mathrm{C}_{4}$ strains, France, 2012. Emerg Infect Dis. 2014;20(8):1343-6. DOI: 10.3201/eid2008.131858 PMID: 25061698

35. SolomonT, LewthwaiteP, PereraD, CardosaMJ, McMinnP, OoiMH. Virology, epidemiology, pathogenesis, and control of enterovirus 71.Lancet Infect Dis. 2010;10(11):778-90. DOI: 10.1016/S1473-3099(10)70194-8 PMID: 20961813

36. LiuCC, ChouAH, LienSP, LinHY, LiuSJ, ChangJY, et al.

Identification and characterization of a cross-neutralization epitope of Enterovirus 71. Vaccine. 2011;29(26):4362-72. DOI: 10.1016/j.vaccine.2011.04.010 PMID: 21501643

37. MizutaK, AbikoC, MurataT, MatsuzakiY, ItagakiT, SanjohK, et al. Frequent importation of enterovirus 71 from surrounding countries into the local community of Yamagata, Japan,

between 1998 and 2003. J Clin Microbiol. 2005;43(12):6171-5. DOI: $10.1128 / J C M .43 .12 .6171-6175.2005$ PMID: 16333123

38. HenquellC, MirandA, RichterJ, Schuffeneckerl, BöttigerB, DiedrichS, et al. Phylogenetic patterns of human coxsackievirus $B_{5}$ arise from population dynamics between two genogroups and reveal evolutionary factors of molecular adaptation and transmission. J Virol. 2013;87(22):12249-59. DOI: $10.1128 / J V I .02075-13$ PMID: 24006446

39. FischerTK, NielsenAY, SydenhamTV, AndersenPH, AndersenB, MidgleySE. Emergence of enterovirus 71 C4a in Denmark, 2009 to 2013. Euro Surveill. 2014;19(38):20911. DOI: 10.2807/15607917.ES2014.19.38.20911 PMID: 25306878 